\title{
Conservación
}

\section{Los mamíferos del Área Reservada para la Recreación y Educación Ecológica San Juan del Monte, Las Vigas de Ramírez, Veracruz, México}

\author{
Mammals of Recreation and Ecological Education Reserved Area San Juan del Monte, \\ Las Vigas de Ramírez, Veracruz, Mexico \\ Helxine Fuentes-Moreno ${ }^{\mathrm{a}, *}$, Aida Trejo-Ortiz ${ }^{\mathrm{b}}$ y Fernando A. Cervantes ${ }^{\mathrm{c}}$ \\ ${ }^{a}$ Compañía Minera Cuzcatlán, Km 42.5 carretera Oaxaca-Puerto Ángel, San José del Progreso, 71250 Ocotlán, Oaxaca, México \\ b Iturbide Núm. 11, 71256 San Bartolo Coyotepec, Oaxaca, México \\ ${ }^{\mathrm{c}}$ Departamento de Zoología, Instituto de Biología, Universidad Nacional Autónoma de México, Apartado postal 70-153, 04510 Ciudad de México, México
}

Recibido el 9 de enero de 2017; aceptado el 31 de mayo de 2017

Disponible en Internet el 26 de noviembre de 2017

\begin{abstract}
Resumen
El Área Reservada para la Recreación y Educación Ecológica San Juan del Monte se ubica en el municipio de las Vigas de Ramírez, Veracruz, México, es importante por la vegetación primaria de pináceas que posee, y porque históricamente ha sido clave en el conocimiento de los mamíferos del estado de Veracruz; sin embargo, previo a este trabajo no contaba con un listado de mastofauna. El trabajo de campo realizado entre $2002-2005$ y abril de 2007 comprendió métodos directos e indirectos de registro y la revisión de literatura. Se reportan 36 especies agrupadas en 15 familias y 7 órdenes; 7 especies son endémicas de México, 12 son compartidas con Norte- y Sudamérica, 11 solamente con Norteamérica y una con Sudamérica. Tres especies se encuentran en alguna categoría de riesgo mexicana. Mediante el trabajo de campo se obtuvieron registros de 18 especies y se destacan 4 nuevos registros para la localidad, la literatura hace referencia a 31 especies, en 8 de las cuales, Las Vigas es la localidad del holotipo. Esta mastofauna representa el 19\% de la total de Veracruz y el 31.5\% de la región montañosa. La riqueza puede deberse a su ubicación en la Faja Volcánica Transmexicana que genera características ecológicas y geográficas particulares.
\end{abstract}

(C) 2017 Universidad Nacional Autónoma de México, Instituto de Biología. Este es un artículo Open Access bajo la licencia CC BY-NC-ND (http://creativecommons.org/licenses/by-nc-nd/4.0/).

Palabras clave: Área natural protegida; Conservación; Inventario; Localidad tipo; Taxonomía

\section{Abstract}

The Recreation and Ecological Education Reserved Area San Juan del Monte is located in the municipality of Vigas de Ramírez, Veracruz, México, and it is important due to its primary vegetation and it historically has been a key regarding the knowledge of mammals in the state of Veracruz; however, previous to this work there was no taxonomic list of its mastofauna. Field work was done between 2002-2005 and April, 2007 including direct and indirect methods and the review of specialized literature. Thirty six species grouped into 15 families and 7 orders are reported; 7 species are endemic to Mexico, 12 are shared with North and South America, 11 are only shared with North America and only 1 is shared with South America. Three species are listed in a Mexican risk category. Through fieldwork, records of 18 species were obtained and a highlight of 4 new records for the locality, literature refers to 31 species, 8 for which Las Vigas is the holotype locality. This mammal set of species equals $19 \%$ of mammal species for Veracruz and $31.5 \%$ just for the mountains of this state. The species richness of the San Juan del Monte area may be due to its geographical location on the Transmexican Volcanic Belt which has distinctive ecological and geographical features.

(C) 2017 Universidad Nacional Autónoma de México, Instituto de Biología. This is an open access article under the CC BY-NC-ND license (http://creativecommons.org/licenses/by-nc-nd/4.0/).

Keywords: Protected natural area; Conservation; Inventory; Type locality; Taxonomy

\footnotetext{
* Autor para correspondencia.

Correo electrónico: hfuentes@mincuzcatlan.com (H. Fuentes-Moreno).

La revisión por pares es responsabilidad de la Universidad Nacional Autónoma de México.
} 


\section{Introducción}

Las áreas naturales protegidas son claves en la conservación de las comunidades biológicas (Primack y Ros, 2000) y una modalidad de ellas es la destinada a la recreación y educación ecológica. El Área Reservada para la Recreación y Educación Ecológica San Juan del Monte, de carácter estatal, se encuentra en esta categoría, y tiene como objetivos la investigación y práctica para capacitar en materia de ecología mediante programas educativos a maestros rurales, niños, campesinos, estudiantes y en general a todas aquellas personas cuya actividad o interés requieran de una educación ecológica fundamental (Vargas, Escobar, de la Maza y del Pont, 2002). La importancia ecológica de San Juan del Monte radica en las grandes extensiones de bosque de pino que alberga y por consiguiente los servicios ambientales que proporciona: retención de agua, regulación del clima y refugio de la flora y la fauna (Vázquez-Torres, CarvajalHernández y Aquino-Zapata, 2010). Sin embargo, en el caso de la fauna, y de los mamíferos en particular, se desconoce cuál es su composición de especies. Previo a este trabajo no existía ningún listado publicado del sitio, a pesar de que Las Vigas, municipio en donde se encuentra San Juan del Monte, ha sido clave en la descripción de nuevas especies.

Es por esto que, considerando que los inventarios biológicos deben ser la base en la toma de decisiones de manejo, conservación y planeación de las áreas naturales protegidas (Llorente, Luna, Soberón y Bojórquez, 1994), se realizó este listado de los mamíferos silvestres del Área Reservada para la Recreación y Educación Ecológica San Juan del Monte, para contribuir al conocimiento y planeación de su aprovechamiento sustentable.

\section{Materiales y métodos}

El Área Reservada para la Recreación y Educación Ecológica San Juan del Monte comprende 609.62 ha y se encuentra ubicada en el municipio de Las Vigas de Ramírez, en el estado de Veracruz, México, en las coordenadas $19^{\circ} 37^{\prime} 53.4^{\prime \prime} \mathrm{N}, 97^{\circ} 7^{\prime} 0.1^{\prime \prime} \mathrm{O}$; $19^{\circ} 37^{\prime} 47.6^{\prime \prime} \mathrm{N}, 97^{\circ} 6^{\prime} 15.8^{\prime \prime} \mathrm{O} ; 1^{\circ} 36^{\prime} 37.4^{\prime \prime} \mathrm{N}, 97^{\circ} 6^{\prime} 15.8^{\prime \prime} \mathrm{O}$; $19^{\circ} 36^{\prime} 27.3^{\prime \prime} \mathrm{N}, 97^{\circ} 5^{\prime} 37.7^{\prime \prime} \mathrm{O} ; 1^{\circ} 36^{\prime} 0.38^{\prime \prime} \mathrm{N}, 97^{\circ} 5^{\prime} 38.67^{\prime \prime} \mathrm{O}$; $19^{\circ} 36^{\prime} 3.2^{\prime \prime} \mathrm{N}, 97^{\circ} 7^{\prime} 15.9^{\prime \prime} \mathrm{O}$ (fig. 1). Colinda al norte con la ciudad de Las Vigas, al sur con el ejido de Toxtlacoaya, al oriente con los lotes del predio San Juan del Monte y al poniente con el ejido de Las Vigas (Vargas et al., 2002). Las partes altas, entre los 2,500-2,750 m, son terrenos abruptos con sierras volcánicas alternando con llanuras y con presencia notable de partes rocosas también conocidas localmente como «malpais»». El clima es templado subhúmedo $\mathrm{C}(\mathrm{w} 1)$, es decir, el más seco de los templados y con nublados frecuentes ya que recibe la humedad proveniente del golfo de México. Los inviernos son fríos, con largos periodos de neblinas y las heladas son usuales en los primeros meses del año. Los veranos son frescos, con lluvias abundantes y noches despejadas. La temperatura promedio anual es de entre 12 y $18^{\circ} \mathrm{C}$, con una temperatura del mes más frío de $-3{ }^{\circ} \mathrm{C}$, mientras que la temperatura del mes más caliente es de $22^{\circ} \mathrm{C}$. La precipitación media anual va de los 200 a los $1,800 \mathrm{~mm}$ y la precipitación en el mes más seco va de los 0

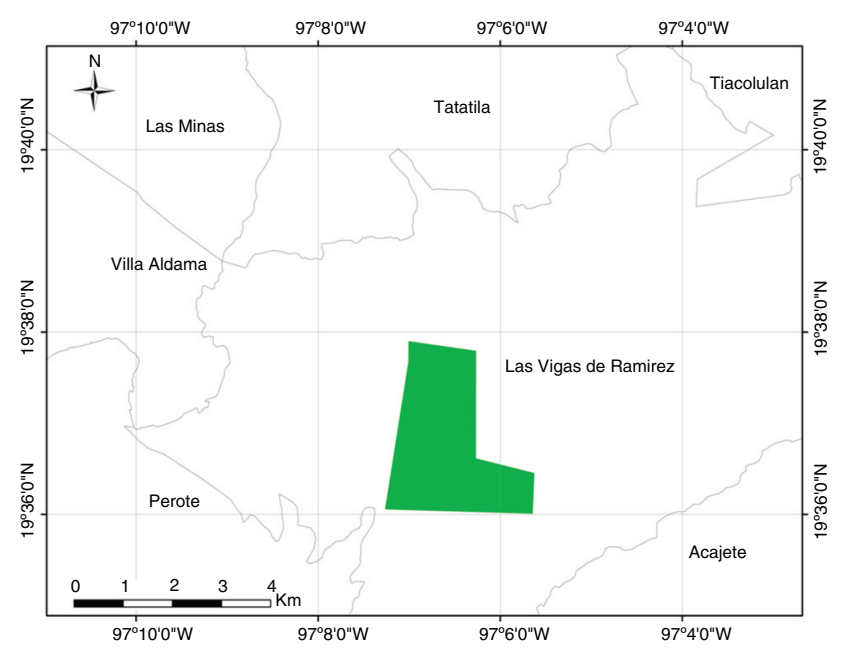

Figura 1. Localización geográfica del Área Reservada para la Recreación y Educación Ecológica San Juan del Monte, Las Vigas de Ramírez, Veracruz, México.

a los $400 \mathrm{~mm}$ (García, 2004). No se presentan escurrimientos importantes, solamente arroyos intermitentes.

La formación vegetal característica de la región es bosque de coníferas de clima templado con suelos de tipo volcánico y ácido. Se reconocen 2 tipos de bosque, el de pino teocote (Pinus teocote) y el bosque mixto con pinos ( $P$. patula y $P$. pseudotrobus) y encinos (Quercus spp.), alnus (Alnus jorullensis) y madroño (Arbutus xalapensis). En las partes bajas se presentan pastizales naturales e inducidos para el pastoreo de ganado y hay abundancia de gramíneas amacolladas conocidas como «zacatones». En el estrato medio se encuentran algunas especies de arbustos (Baccharis conferta, Pertnettia cicliata, Acaena elongata y Buddleia sp.) y en el estrato bajo algunos zacatones y helechos (Alchemilla pectinata, Archibaccharis androgyna, Muhlenbergia macroura, Pteridium aquilinum, Brachypodium mexicanum, Triniochloa stipoides y Piptochaetium pirescens).

El trabajo de campo abarcó todos los meses entre los años 2002-2005 y abril 2007 y se realizó a una altitud promedio de $2,420 \mathrm{~m}$. Para la captura de roedores y musarañas se colocaron al atardecer 20 trampas Sherman cebadas con una mezcla de avena, vainilla y crema de cacahuate durante 4 noches cada mes. Para las tuzas se colocaron 4 trampas de cepo. Para los murciélagos se colocaron al atardecer (18:00 h) en bordes de fragmentos de vegetación, redes de niebla de $6 \mathrm{~m}$ de largo, $2.5 \mathrm{~m}$ de alto y $30 \mathrm{~mm}$ de abertura de malla, a una altura de $3 \mathrm{~m}$ del suelo, las cuales permanecieron abiertas hasta las 12 de la noche.

El listado fue complementado con la identificación de restos de mamíferos recuperados de excrementos de carnívoros (Aranda, 2000; Murie, 1974); los cuales se obtuvieron en búsquedas libres cerca de los sitios donde se colocaron trampas Sherman y redes de niebla y a los lados de los caminos, también se recopiló información en pláticas informales con los pobladores y se observaron pieles y partes de ejemplares obtenidos en la cacería.

Los ejemplares colectados fueron medidos utilizando una regla o una cinta métrica, ambas graduadas en milímetros ( $\mathrm{mm}$ ). Se obtuvieron sus medidas somáticas convencionales: longitud 
total (LT), de la punta de la nariz a la punta de la cola vertebral; longitud de cola vertebral $(\mathrm{CV})$, de la base de la cola hasta la última vértebra de la cola; longitud de pata derecha trasera (PT), del talón a la punta de la uña más larga; longitud de la oreja derecha $(\mathrm{O})$, de la base a la parte más alejada del pabellón auditivo. En el caso de los murciélagos también se obtuvo la longitud del antebrazo ( $\mathrm{AB})$, que es la longitud total del radio y el cúbito. Los ejemplares fueron pesados con un dinamómetro graduado en gramos (g). Algunos especímenes fueron preparados en piel y esqueleto, guardando una muestra de tejidos y ectoparásitos, siempre que fue posible (Hafner, Hafner y Hafner, 1984). Todo el material mastozoológico que se obtuvo se encuentra depositado en la Colección Nacional de Mamíferos (CNMA) del Instituto de Biología, de la Universidad Nacional Autónoma de México (apéndice).

Se buscaron registros de mamíferos para el área de estudio en artículos científicos, memorias de congresos, libros y, particularmente, la monografía de los mamíferos de Veracruz (Hall y Dalquest, 1963), donde una de las localidades importantes de referencia es Las Vigas. En la internet se utilizó el buscador VertNet usando las palabras clave «Las Vigas», «Mamíferos» y «Veracruz»; se revisó también la base de datos de la Colección Nacional de Mamíferos, Instituto de Biología, UNAM. Debido a que muchas publicaciones son antiguas, se revisó la sinonimia de las especies y se confirmaron la nomenclatura y clasificación utilizando los trabajos de Gaona, González-Christen y López-Wilchis (2003), Guevara, Cervantes y Sánchez-Cordero (2015), Helgen, Cole, Helgen y Wilson (2009), Ramírez-Pulido, González-Ruiz, Gardner y Arroyo-Cabrales (2014) y Villa y Cervantes (2003).

\section{Resultados}

Mediante el trabajo de campo se obtuvieron recolectas y registros de 18 especies, entre los cuales destacan 4 nuevos registros para la localidad: armadillo (Dasypus novemcinctus mexicanus), mapache (Procyon lotor hernandezii), musaraña (Cryptotis parva pueblensis) y murciélago (Myotis californicus mexicanus); uno más, el gato montés (Lynx rufus escuinapae), había sido observado (Hall y Dalquest, 1963) pero no existían recolectas.

Los resultados del trabajo de campo y la revisión de la literatura arrojan un total de 36 especies agrupadas en 15 familias y 7 órdenes que componen la mastofauna del Área Reservada para la Recreación y Educación Ecológica San Juan del Monte. Siete especies son endémicas de México, destacando 4 roedores (Sciurus oculatus oculatus, Peromyscus beatae beatae, Neotomodon alstoni y Cratogeomys merriami estor). Por su afinidad biogeográfica, 12 especies son compartidas con Norte- y Sudamérica, 11 solamente con Norteamérica y una es compartida solamente con Sudamérica (tabla 1). Tres de las especies se encuentran en categorías de riesgo definidas por el gobierno mexicano (Semarnat, 2010): una ardilla (Sciurus oculatus oculatus) y una musaraña (Sorex saussurei veraecrucis), en la categoría de sujeta a protección especial y una musaraña ( $S$. macrodon) como amenazada.
El orden con mayor riqueza específica y diversidad de hábitos de vida fue Rodentia con 13 especies, las que incluyen ardillas arborícolas, ratones y ratas terrestres, y tuzas hipogeas; le siguen los murciélagos, Chiroptera, donde 7 de las 8 especies reportadas aprovechan el nicho trófico de la insectivoría; finalmente, los carnívoros se encuentran representados por 7 especies cuya alimentación va desde prácticamente la omnivoría, como el coyote (Canis latrans), hasta la carnivoría estricta como el gato montés (Lynx rufus).

En la literatura se hace referencia a 31 especies que han sido recolectadas en Las Vigas de Ramírez e incluso 8 taxones la tienen como localidad tipo (tabla 2).

\section{Discusión}

Las recolectas más antiguas de Las Vigas fueron publicadas por Ward (1891) y pertenecen al murciélago Vesperugo veraecrucis, actualmente Perimyotis subflavus veraecrucis, y al murciélago Centurio minor, actualmente Centurio senex senex; en la localidad del primero, el autor hace referencia a Las Vegas, mientras que en el segundo la localidad es cerro de los Pájaros, Las Vegas. Posteriormente Hall y Dalquest (1963) reconocen, aunque con cautela, como Las Vigas a ambas localidades. El registro más reciente pertenece a Spermophilus (actualmente Otospermophilus) variegatus variegatus (FuentesMoreno, Trejo-Ortiz y Mateos, 2010) y es importante debido a que esta ardilla de tierra o «moto» como le llaman en Las Vigas, fue incluida en los mamíferos del estado de Veracruz hasta el último listado publicado (González, 2011).

La presencia del murciélago Centurio senex senex, único taxón de afinidad neotropical, probablemente se trate de un registro anómalo, similar al encontrado por Uhart y López-Vidal (2008) en el Estado de México. A la fecha no se cuenta con otro registro de este murciélago en el municipio y no es factible su presencia en el área protegida.

Los mamíferos registrados en este trabajo y en la revisión de literatura especializada comprenden el $19 \%$ de las especies con distribución en Veracruz (192; González, 2011). Sin embargo, si consideramos únicamente la región montañosa, a la que pertenece el área de estudio, la riqueza mastofaunística alcanza el $31.5 \%$ de las 114 especies registradas en esta región, la tercera con mayor riqueza de mamíferos en el estado (González, 2011).

Este porcentaje de diversidad toma una mayor importancia al considerar que San Juan del Monte cuenta con una extensión de 609.6 ha y Las Vigas 3,103.63 ha, que representan el $0.1 \%$ del territorio estatal (Sefiplan, 2015; Vargas et al., 2002). Las Vigas de Ramírez es considerado también como uno de los municipios veracruzanos que cuenta con vegetación primaria en buen estado por lo que es importante su conservación (Márquez y Márquez, 2009).

La riqueza mastofaunística reportada en este trabajo resulta proporcional comparándola con otras áreas de clima similar, como son el Parque Nacional Cofre de Perote, donde se reportan 49 especies de mamíferos silvestres, aunque este último cuenta con 3 tipos de vegetación (bosque de coníferas, oyamel y páramo de altura), definidos por el intervalo altitudinal que va de los 2,400 a los 4,250 m snm y su extensión es mayor (11,700 ha; 
Tabla 1

Listado taxonómico de los mamíferos del Área Reservada para la Recreación y Educación Ecológica San Juan del Monte, Las Vigas de Ramírez, Veracruz.

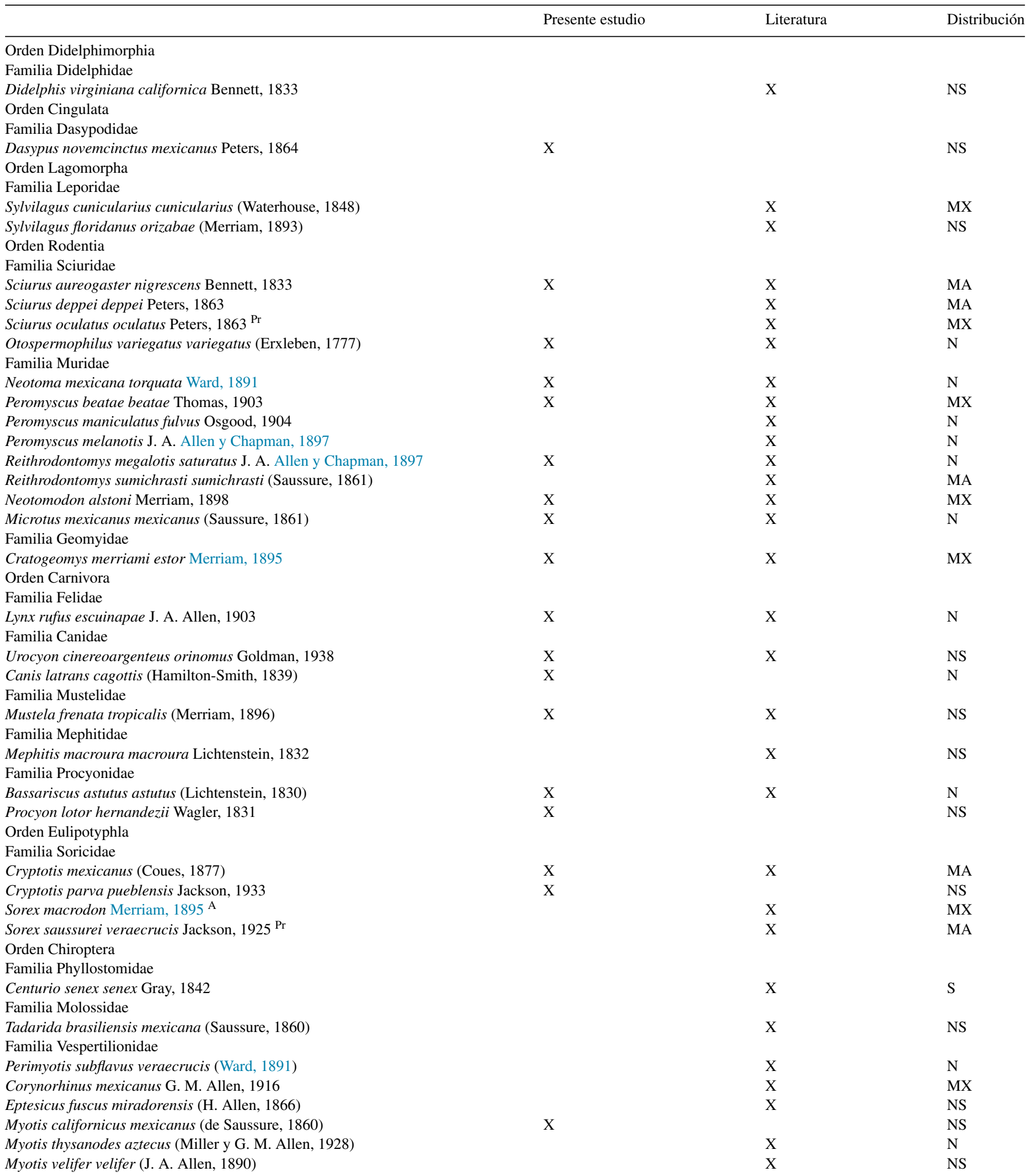

A: amenazada (Semarnat, 2010); MA: endémica de Mesoamérica; MX: endémicas de México; N: compartida con Norteamérica; NS: compartida con Norte- y Sudamérica; Pr: protección especial; S: compartida con Sudamérica (Villa y Cervantes, 2003). 
Tabla 2

Holotipos de mamíferos silvestres de Las Vigas, Veracruz.

\begin{tabular}{|c|c|c|}
\hline Nombre con el que se describe a la especie & Referencia original & Nombre actual \\
\hline Sciurus niger melanonotus & Thomas, 1890 & Sciurus oculatus oculatus Peters, 1863 \\
\hline Centurio minor & Ward, 1891 & Centurio senex senex Gray, 1842 \\
\hline Vesperugo veraecrucis & Ward, 1891 & Perimyotis subflavus veraecrucis (Ward, 1891) \\
\hline Cratogeomys estor & Merriam, 1895 & Cratogeomys merriami estor Merriam, 1895 \\
\hline Peromyscus melanotis & Allen y Chapman, 1897 & Peromyscus melanotis J. A. Allen y Chapman, 1897 \\
\hline Reithrodontomys saturatus & Allen y Chapman, 1897 & Reithrodontomys megalotis saturatus J. A. Allen y Chapman, 1897 \\
\hline Sciurus aureogaster frumentor & Nelson, 1899 & Sciurus aureogaster nigrescens Bennett, 1833 \\
\hline Lepus verae-crucis & Nelson, 1909 & Sylvilagus cunicularius cunicularius (Waterhouse, 1848) \\
\hline
\end{tabular}

(Semarnat, 2015a); así como el Parque Nacional Pico de Orizaba, el cual triplica en extensión a San Juan, ya que abarca 19,750 ha y tiene reportada una riqueza de 45 especies de mamíferos distribuidos en vegetación de pináceas, encinares, oyamel y páramo de altura entre los 3,038 y los 5,636 m snm (Semarnat, 2015b). Las 3 áreas comparten 16 especies y aquellas que se registraron en el Cofre de Perote y/o el Pico de Orizaba podrían estar adaptadas a una vegetación especifica o ser el resultado de la historia geológica del sitio.

Biogeográficamente, la zona es influenciada por la cuenca oriental de la Faja Volcánica Transmexicana al ubicarse al norte del Cofre de Perote, de ahí el componente de endemismos mexicanos o mesoamericanos reportado en este trabajo, lo que representa un tercio de las especies (33\%), de las cuales 7 corresponden a roedores. Esta región ha sido identificada como fuente de especiación (Fernández, Cervantes y Hafner, 2012) y en otros casos favorece una alta diversidad debido a su historia biogeográfica y características ecológicas que incluyen varios hábitats y gradientes altitudinales (Hafner et al., 2005).

Sin embargo, las amenazas por el cambio de uso de suelo y reducción del hábitat por actividades antropogénicas como la agricultura, ganadería y urbanización son factores que a través de los años han amenazado de forma acelerada el territorio veracruzano (Hernández, Delfín y Sosa, 2011), por lo que en el transcurso de más de una década, desde el inicio de las recolectas de este trabajo hasta la fecha, probablemente han influido en la reducción de las poblaciones de algunas especies de mamíferos y sus áreas de distribución.

Las especies más vulnerables y que probablemente se han visto afectadas por esta situación dentro de la Reserva para la Recreación y Educación Ecológica San Juan del Monte son las pertenecientes a los órdenes Rodentia y Eulipotyphla, ya que estos organismos ocupan áreas de distribución geográfica altamente restringida y cuentan con una capacidad de desplazamiento relativamente limitada (Hernández et al., 2011), situación que podría aplicar a $S$. oculatus oculatus, $S$. saussurei veraecrucis y $S$. macrodon, especies en categoría de riesgo en San Juan del Monte.

Existen también especies que se adaptan e incluso se benefician con la presencia de actividades antropogénicas como los tlacuaches y comadrejas, aunque no se puede asegurar el incremento y salud de sus poblaciones.

Debido a que este es el primer trabajo de mamíferos en el sitio, no existen datos previos ni posteriores con los que se pueda equiparar la situación actual de su mastofauna. Asimismo, el problema principal de esta reserva es el poco conocimiento que se tiene de ella (Vázquez-Torres et al., 2010), por lo que es difícil precisar las amenazas y el estado actual de conservación de sus especies.

Finalmente, San Juan del Monte es un área biológicamente importante debido a su rica composición mastofaunística, resultado de su ubicación y de su intrincada historia biogeográfica. Esta mastofauna ha contribuido con múltiples servicios ambientales a la conservación de su extensión boscosa y al sostenimiento de la población humana. Ahora es necesaria la difusión de esta información para poder generar una educación ambiental y la utilización responsable de estos recursos naturales, destacando que la intención de establecer esta área natural protegida es para preservar las zonas boscosas conservadas con que cuenta e informar a todas aquellas personas cuya actividad o interés requieran de educación ambiental. Este listado, por lo tanto, contribuye a la educación, manejo y conservación del área.

\section{Agradecimientos}

A la Coordinación Estatal de Medio Ambiente, Xalapa, Veracruz, la Universidad Veracruzana, Facultad de Ciencias Biológicas y Agropecuarias-Peñuela y Compañía Minera Cuzcatlán. A los maestros Yolanda Hortelano, Rafael Flores y Flora H. Zitácuaro por sus acertadas sugerencias al manuscrito. A los guardabosques Don León, Margarito Becerra y Benjamín Becerra. A Jorge Martínez por su ayuda en la preparación de ejemplares y a la familia Fuentes por su apoyo moral y económico. J. Franco amablemente ayudó en la traducción del resumen al inglés.

\section{Apéndice. Ejemplares de mamíferos silvestres recolectados en el Área Reservada para la Recreación y Educación Ecológica San Juan del Monte, Las Vigas de Ramírez, Veracruz, y depositados en la Colección Nacional de Mamíferos del Instituto de Biología, UNAM.}

Sciurus aureogaster nigrescens Bennett, 1833. Ejemplares colectados (4): CNMA 42788, 42790, 42791, 42792. Otospermophilus variegatus variegatus (Erxleben, 1777). Ejemplares colectados (1): CNMA 45036. $0^{7}, 500-240-55-20 \equiv 1000$. Neotoma mexicana torquata Ward, 1891. Ejemplares colectados (2): CNMA 42804, 42805. Peromyscus beatae beatae Thomas, 1903. Ejemplares colectados (1): CNMA 44805. $0^{7}, 185-100-$ $21-20 \equiv 18$. Reithrodontomys megalotis saturatus $\mathrm{J}$. A. Allen y 
Chapman, 1897. Ejemplares colectados (10): CNMA 44806, 44807, 44808, 44809, 44810, 44811, 44812, 44813, 45039, 45040. Medidas promedio (intervalo) de 907, 1o. LT: 147.3 (130-160), CV: 76 (62-85), PT: 18 (14-20), O: 16 (13-21), peso 13.4 (10-17). Neotomodon alstoni Merriam, 1898. Ejemplares colectados (18): CNMA 42806, 42807, 42808, 42809, 42810, 44795, 44796, 44797, 44798, 44799, 44800, 44801, 44802, 44803, 44804, 45700, 45701, 45702. Medidas promedio (intervalo) de $80^{7}$ y 5\%. LT: 196.6 (161-280), CV: 82.8 (69-95), PT: 24.6 (22-27), O: 22.2 (17-27), peso 38.2 (25-52). Microtus mexicanus mexicanus (Saussure, 1861). Ejemplares colectados (5): CNMA 42800, 42801, 42802, 42803, 45699. 10`, 145-34-20-19=30. Cratogeomys merriami estor Merriam, 1895. Ejemplares colectados (9): CNMA 42793, 42794, 42795, 42797, 42798, 44792, 44793, 44794, 45037. Medidas promedio (intervalo) de $10^{7}$ y 3 o. LT: 272.8 (225-232), CV: 40.8 (17-84), PT: 37.5 (33-43), O: 6 (4-8), peso 672.3 (192-1500). Lynx rufus escuinapae J. A. Allen, 1903. Ejemplares colectados (1): CNMA 47897. Mustela frenata tropicalis (Merriam, 1896). Ejemplares colectados (1): CNMA 42786. Bassariscus astutus astutus (Lichtenstein, 1830). Ejemplares colectados (2): CNMA 44790, 45034. Procyon lotor hernandezii Wagler, 1831. Ejemplares colectados (1): CNMA 45035. Cryptotis mexicanus (Coues, 1877). Ejemplares colectados (11): CNMA 42773, 42774, 42775, 42776, 42777, 42778, 42779, 42780, 42781, 44786, 44787. Medidas de 1o: 100-31-13-5=9; 1?: 90-25-13-2=?. Cryptotis parva pueblensis Jackson, 1933. Ejemplares colectados (2): CNMA 42782, 42783. Myotis californicus mexicanus (de Saussure, 1860). Ejemplares colectados (2): CNMA 44788, 44789. Medidas de 10 : 72-35-7-12-30=5 y 1 : 78-37-6-14-31=25.

\section{Referencias}

Allen, J. A. y Chapman, F. M. (1897). On a collection of mammals from Jalapa and Las Vigas, state of Vera Cruz (1897). Bulletin of the American Museum of Natural History, 9, 197-208.

Aranda, M. (2000). Huellas y otros rastros de los mamíferos grandes y medianos de México. Xalapa, Veracruz: Instituto de Ecología, A.C.

Fernández, J. A., Cervantes, F. A. y Hafner, M. A. (2012). Molecular systematic and biogeography of the Mexican endemic kangaroo rat, Dipodomys phillipsii (Rodentia: Heteromyidae). Journal of Mammalogy, 93, 560-571.

Fuentes-Moreno, H., Trejo-Ortiz, A. y Mateos, M. L. (2010). Otospermophilus variegatus, una ardilla olvidada de Veracruz. In En X Congreso Nacional y I Congreso Latinoamericano de Mastozoología. pp. 120. Guanajuato, México: Asociación Mexicana de Mastozoología, A.C.

Gaona, S., González-Christen, A. y López-Wilchis, R. (2003). Síntesis del conocimiento de los mamíferos silvestres del Estado de Veracruz México. Revista de la Sociedad Mexicana de Historia Natural, 3. ${ }^{a}$ Época, 1, 91-108.

García, E. (2004). Modificaciones al sistema de clasificación climática de Koeppen. México D.F.: Instituto de Geografía, Universidad Nacional Autónoma de México.

González, C. A. (2011). Mamíferos: distribución, endemismo y estado de conservación. En A. A. Cruz, (Coordinador y Editor general), La biodiversidad en Veracruz: estudio de estado (pp. 579-592). Xalapa, Veracruz: Comisión para el Conocimiento y Uso de la Biodiversidad, Gobierno del Estado de Veracruz, Universidad Veracruzana, Instituto de Ecología, A.C.

Guevara, L., Cervantes, F. A. y Sánchez-Cordero, V. (2015). Riqueza, distribución y conservación de los topos y las musarañas (Mammalia, Eulipotyphla) de México. Therya, 6, 43-68.
Hafner, D. J., Hafner, J. C. y Hafner, M. S. (1984). Skin-plus-skeleton preparation as the standard mammalian museum specimen. Curator, 27, $141-145$.

Hafner, M. S., Light, J. E., Hafner, D. J., Brant, S. V., Spradling, T. A. y Demastes, J. W. (2005). Cryptic species in the Mexican pocket gopher Cratogeomys merriami. Journal of Mammalogy, 86, 1095-1108.

Hall, R. y Dalquest, W. W. (1963). The mammals of Veracruz. University of Kansas Publication, Museum of Natural History, 14, 165-362.

Helgen, K. M., Cole, F. R., Helgen, L. E. y Wilson, D. E. (2009). Generic revision in the holartic ground squirrel genus Spermophilus. Journal of Mammalogy, 90, 270-305.

Hernández, H. A., Delfín, A. C. y Sosa, F. V. J. (2011). Análisis regional de las principales amenazas para las especies de mamíferos incluidas en la NOM059-SERMARNAT-2001. En A. Cruz (Coordinador y Editor general), La biodiversidad en Veracruz: estudio de estado (pp. 629-645). Xalapa, Veracruz: Comisión para el Conocimiento y Uso de la Biodiversidad, Gobierno del Estado de Veracruz, Universidad Veracruzana, Instituto de Ecología, A.C.

Llorente, B. J., Luna, V. I., Soberón, M. J. y Bojórquez, T. L. (1994). Biodiversidad su inventario y conservación: teoría y práctica en la taxonomía alfa contemporánea. En B. J. Llorente e y I. Luna (Eds.), Taxonomía biológica. (pp. 507-521). México D.F.: Fondo de Cultura Económica.

Márquez, R. W. y Márquez, R. J. (2009). Municipios con mayor biodiversidad en Veracruz. Foresta Veracruzana, 11, 43-50.

Merriam, C. H. (1895). Monographic revision of the pocket gophers, family Geomyidae, exclusive of the species of Thomomys. North American Fauna, 8, 1-258.

Murie, O. J. (1974). A field guide to animal tracks. Boston: Houghton Mifflin Company.

Nelson, E. W. (1899). Revision of the squirrels of Mexico and Central America. Proceedings of the Washington Academy of Sciences, 1, 15-110.

Nelson, E. W. (1909). The rabbits of North America. North American Fauna, $29,1-314$.

Primack, R. B. y Ros, J. (2000). Introducción a la biología de la conservación. Barcelona: Editorial Ariel, S.A.

Ramírez-Pulido, J., González-Ruiz, N., Gardner, A. L. y Arroyo-Cabrales, J. (2014). List of recent land mammals of Mexico 2014. Special Publications, Museum of Texas Tech University, 63, 1-73.

Sefiplan (Secretaría de Finanzas y Planeación del Estado de Veracruz). (2015). Sistema de Información Municipal. Cuadernillos Municipales, 2015. Las Vigas de Ramírez. México. Recuperado 6 Ago 2016 de: http://ceieg.veracruz.gob.mx/cuadernillos-municipales-2016/

Semarnat (Secretaría de Medio Ambiente y Recursos Naturales). (2010). Norma Oficial Mexicana NOM-059-SEMARNAT-2010, Protección ambientalEspecies nativas de México de flora y fauna silvestres-Categorías de riesgo y especificaciones para su inclusión, exclusión o cambio-Lista de especies en riesgo. México D.F.: Diario Oficial de la Federación.

Semarnat (Secretaría de Medio Ambiente y Recursos Naturales). (2015a). Programa de manejo Parque Nacional Cofre de Perote o Nauhcampatépetl. México. Recuperado 6 Ago 2016 de: http://www.conanp.gob. mx/que_hacemos/pdf/programas_manejo/2015/Libro_Cofre_de_Perote.pdf

Semarnat (Secretaría de Medio Ambiente y Recursos Naturales). (2015b). Programa de manejo Parque Nacional Pico de Orizaba. México. Recuperado 6 Ago 2016 de: http://www.conanp.gob.mx/que_hacemos/ pdf/programas_manejo/2015/Pico_de_Orizaba.pdf

Thomas, O. (1890). On a collection of mammals from Central Vera Cruz Mexico. Proceedings of the Zoological Society of London, 71-76.

Uhart, E. Q. y López-Vidal, J. C. (2008). Registro anómalo en la distribución del murciélago cara de viejo Centurio senex (Chiroptera: Mammalia). Revista Mexicana de Mastozoología, 12, 176-179.

Vargas, M. F., Escobar, M. S., de la Maza, E. R. y del Pont, L. R. M. (2002). Áreas naturales protegidas de México con decretos estatales, Volumen 2. México D.F.: Secretaría de Medio Ambiente y Recursos Naturales, Instituto Nacional de Ecología y Comisión Nacional de Áreas Naturales Protegidas.

Vázquez-Torres, S. M., Carvajal-Hernández, C. I. y Aquino-Zapata, A. M. (2010). Áreas naturales protegidas. En G. B. Benítez y C. R. Welsh (Eds.), Atlas del patrimonio natural, histórico y cultural de Veracruz, 
Vol.1. Patrimonio natural. (pp. 249-274). Xalapa: Comisión del Estado de Veracruz para la Conmemoración de la Independencia Nacional y de la Revolución Mexicana, Gobierno del Estado de Veracruz y Universidad Veracruzana.
Villa, R. B. y Cervantes, F. A. (2003). Los mamíferos de México. México D.F.: Grupo Editorial Iberoamérica.

Ward, H. L. (1891). Descriptions of three new species of Mexican bats. The American Naturalist, 25, 743-753. 